\title{
Review Paper \\ Broccoli and Helicobacter Pylori: A Systematic Review
}

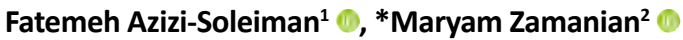

1. Department of Nutrition, School of Health, Arak University of Medical Sciences, Arak, Iran.

2. Department of Epidemiology, School of Health, Arak University of Medical Sciences, Arak, Iran.

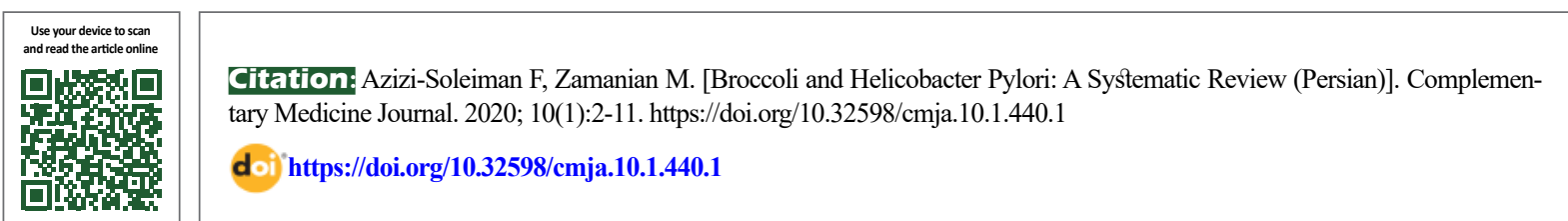

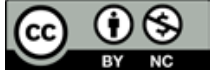

Article Info:

Received: 15 Apr 2019

Accepted: 20 Apr 2019

Available Online: 01 Jun 2020

Keywords:

Helicobacter pylori,

Helicobacter, Broc-

coli, Sulforaphane,

Clinical trial

\section{ABSTRACT}

Objective Pharmacological treatment of Helicobacter pylori (H. pylori) infection is based on the use of at least two antibiotics with a double dose of proton pump inhibitor which results in antibiotic resistance. Anti-helicobacterial activity of sulforaphane-rich broccoli has been evaluated in laboratory studies. This study aimed to systematically review the conducted randomized clinical trials that have examined the effect of broccoli on $\mathrm{H}$. pylori in humans.

Methods This study is a systematic review of randomized clinical trials on the effect of broccoli on $\mathrm{H}$. pylori. The search was conducted in PubMed, OVID, Web of Science, and Scopus databases using the keywords: Helicobacter pylori, broccoli sprouts, H. pylori, randomized clinical trials, and Brassica, without any time limits for studies conducted until 2019. After excluding duplicates, the titles and abstracts of remained articles were evaluated by two researchers and then the related ones were extracted. Next, their full-texts were examined to select the final articles for review. We included clinical trials and excluded those were in the laboratory or animal testing phases or their full-texts were unavailable.

Results Three studies that had met the inclusion criteria were considered for the review. Overall, neither in the articles that reviewed in the present study nor in the articles that did not enter the review process due to unavailability of their full-texts or having a very small sample size, no clear positive effect of broccoli on inhibiting $\mathrm{H}$. pylori infection in humans had been reported.

Conclusion Due to the lack of optimal results from broccoli consumption for the control of H. pylori infection in humans, it is recommended that longer studies with sufficient sample size and appropriate dose of broccoli along with standard treatment be performed in the future.

\section{Extended Abstract}

\section{Introduction}

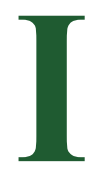

$\mathrm{n}$ developing countries, the incidence of Helicobacter pylori (H. pylori) is $70-80 \%$, while in developed countries it is about $13-50 \%$ of the population [2]. Pharmacological treatment of Helicobacter pylori infection is based on the use of at least two antibiotics with a double dose of proton pump inhibitor. The problem with eradication treatment is the sudden increase in H. pylori's resistance to antibiotics. It seems that the use of herbal compounds reduces the side effects of drugs and causes their better efficiency [5]. The high anti H. pylori activity of Broccoli, Sulforaphane has been shown in in-vitro studies [6]. The aim of this study was to conduct a systematic review of randomized clinical trials that examined the effect of broccoli on human H. pylori infection.

\section{* Corresponding Author:}

Maryam Zamanian, PhD.

Address: Department of Epidemiology, School of Health, Arak University of Medical Sciences, Arak, Iran

Tel: +98 (918) 3497518

E-mail: zamanian.m2015@gmail.com 


\section{Materials and Methods}

In this systematic review, searching was conducted in PubMed, OVID, web of science, and Scopus databases using keywords: Helicobacter pylori, Broccoli sprout, $\mathrm{H}$. pylori, Brassica, and Randomized clinical trial on articles in English published until January 2019. After searching, duplicate articles were deleted. The titles and abstracts of the articles were evaluated by two independent individuals and related articles were selected. Acceptable studies should be of a randomized clinical trial. They should not be in the laboratory or animal testing phases and should have available full text. The full texts of the related articles were then read and the final articles were selected. Figure 1 illustrates the screening process. The information of each study, including the authors' name, the country of study, the number of samples, the year of study, and the results of the study were summarized in Table 1.

\section{Results}

Initially, 48 studies were found by searching databases and using related keywords. After removing duplicate items, 21 studies were evaluated by examining their title and abstract. Finally, only three related studies with the desired criteria were selected for review. These studies were conducted from 2009 to 2015 in three countries: Korea, Japan and Iran (Table 1). In the study conducted by Chang et al. [12] on 89 patients randomly assigned to three treatment groups, broccoli sprout extract containing sulforaphane did not inhibit the H. pylori infection density.

Bahadoran et al. [13] in a study in Iran on 86 type 2 diabetic patients with positive $\mathrm{H}$. pylori randomly assigned to three treatment groups, showed that Broccoli, despite a considerable effect, could not compete with the three-drug treatment. In another rstudy, Yanaka et al. [14] examined $48 \mathrm{H}$. pylori-infected patients in Japan randomly assigned to two groups. Their results showed that Broccoli could reduce H. pylori but could not completely eradicate it. Overall, neither in the articles that reviewed in the present study nor in the articles that did not enter the review process due to unavailability of their full-texts [8-10] or having a very small sample size [11], no clear effect of broccoli on inhibiting H. pylori infection had been reported.

\section{Discussion}

According to the present systematic review study, the consumption of broccoli has no therapeutic effect in control-

Table 1. Specifications of studies on the effect of broccoli on H. pylori infection

\begin{tabular}{|c|c|c|c|c|c|c|}
\hline $\begin{array}{l}\text { ज़ } \\
\frac{\vdots}{0} \\
\text { 菨 }\end{array}$ & Title & 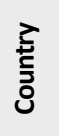 & 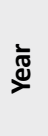 & Samples & Study Groups & Findings \\
\hline
\end{tabular}

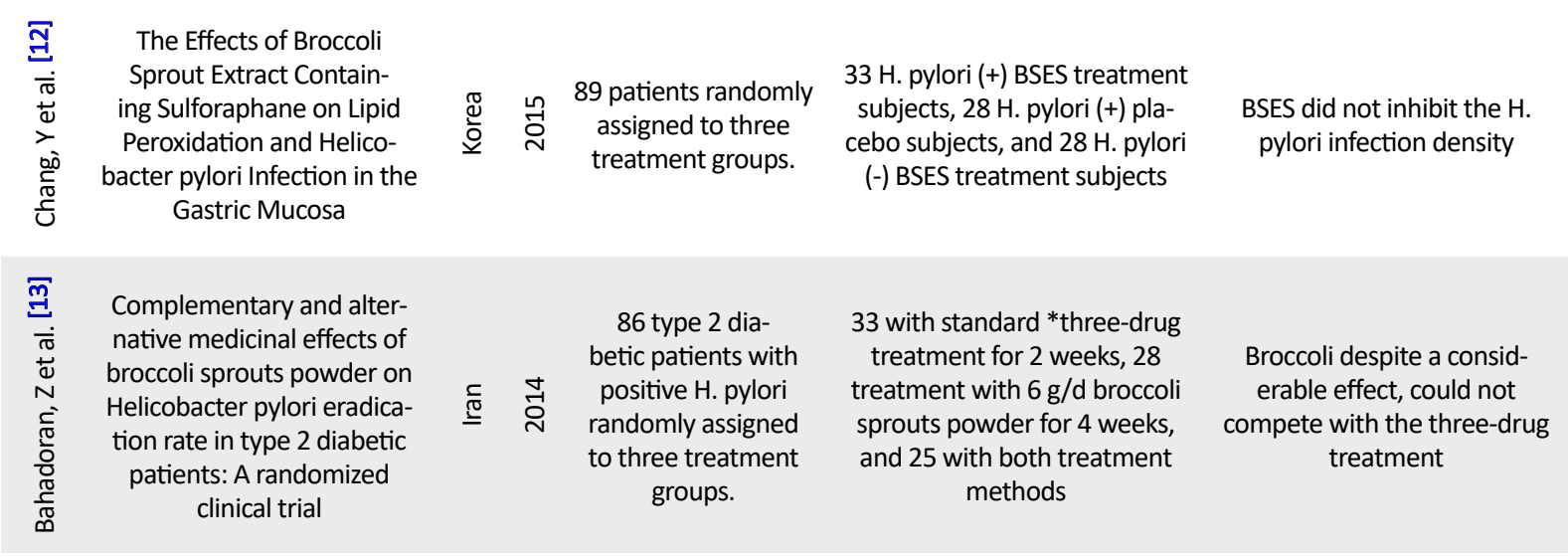

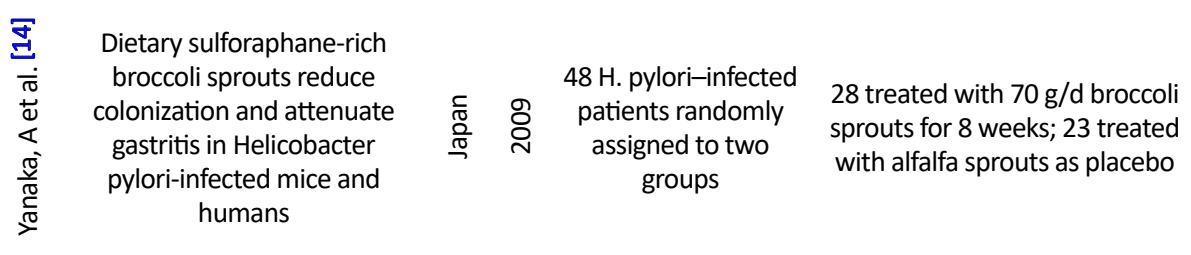

BSES $=$ Broccoli Sprout Extract containing Sulforaphane

*Omeprazole $20 \mathrm{mg}$, clarithromycin $500 \mathrm{mg}$, amoxicillin $1000 \mathrm{mg}$, twice a day for 14 days
Broccoli reduced $\mathrm{H}$.pylori but did not completely eradicate it. 


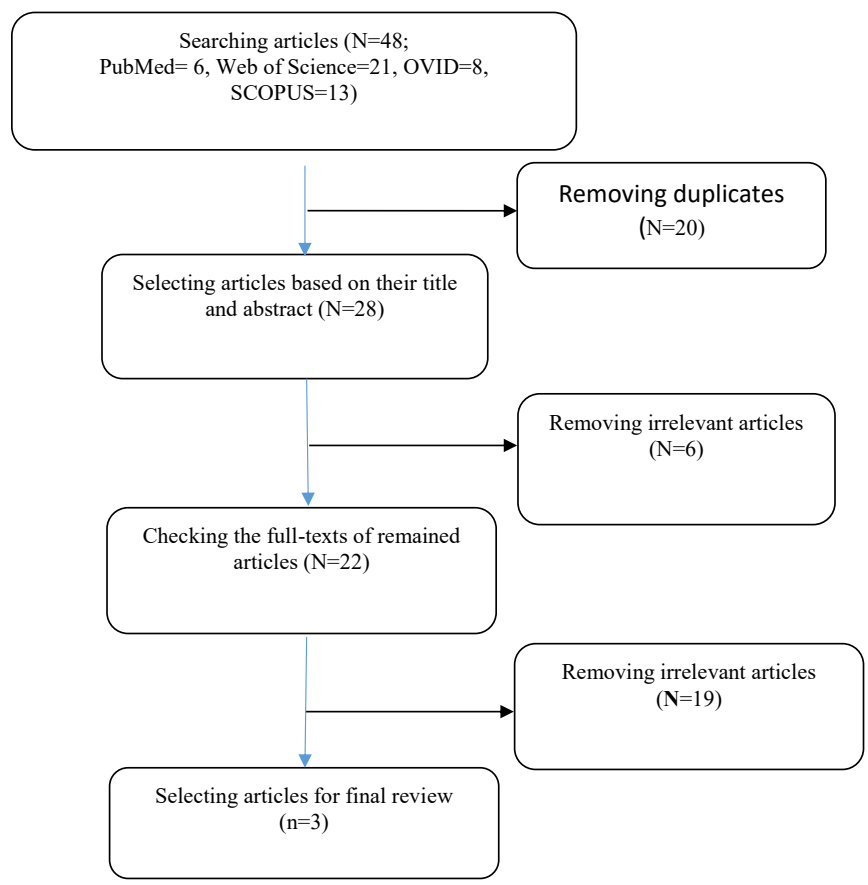

Figure 1. Flowchart of screening process for selecting articles

ling H. pylori infection. There are several biologically active compounds in broccoli, including carotenoids, phylloquinone, antioxidant vitamins, vitamins B1, B2 and selenium, but its main bioactive compound that is responsible for its beneficial effects is sulforaphane [15]. The beneficial effects of this compound on eradication and improvement of the complications of $\mathrm{H}$. pylori infection have been shown in several cellular and animal studies. sulforaphane has a inhibitory effect on H. pylori in human epithelial cells [16].

Studies have shown that the gastric microbiota (e.g. Clostridium spp) affects the severity of H. pylori infection [20]. The reviewed study had not been addressed this issue. The degree of antibiotic resistance of individuals before the study can also affect the results. In the reviewed studies, broccoli extract, powder and sprouts were used in different doses, which in turn can affect the concentration of sulforaphane and other active ingredients of broccoli [21] and have different results on the eradication of $\mathrm{H}$. pylori infection.

\section{Conclusion}

Looking more closely at the studies, it seems that the reason for not seeing the desired result of broccoli in controlling the H. pylori infection is related to the difference in the severity of infection with this bacterium, dose and type of the used broccoli, the duration of study, the use or non-use of concomitant standard treatment, and insufficient sample size. Due to the lack of optimal results from broccoli consumption for the control of H. pylori infection in humans, it is recommended that longer studies with sufficient sample size and appropriate dose of broccoli, along with standard treatment be performed in the future.

\section{Ethical Considerations}

Compliance with ethical guidelines

This is a review article with no human or animal specimens and no need for ethics code.

Funding

Arak University of Medical Sciences financially supported this study.

Authors' contributions

All authors contributed in preparing this paper.

Conflicts of interest

The authors declare no conflict of interest

Acknowledgements

The authors would like to thank Arak University of Medical Sciences for their financial support and cooperation. 


\title{
بروكلى و هليكوباكتر ييلورى: يك مطالعه مرورى منظم
}

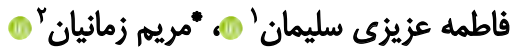 \\ ا. كروه تغذيه، دانشكده بهداشت ، دانشكاه علوميزشكى اراك، اراك، ايران.

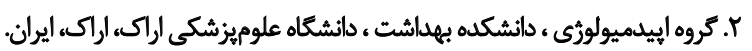

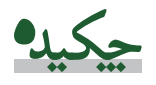

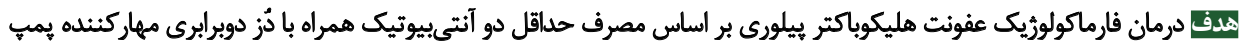

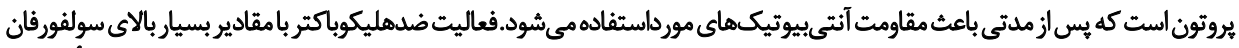

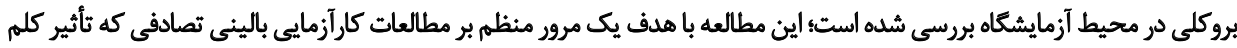

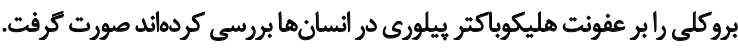

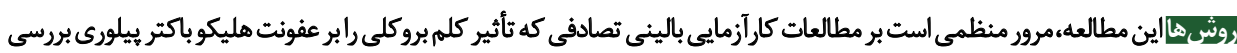

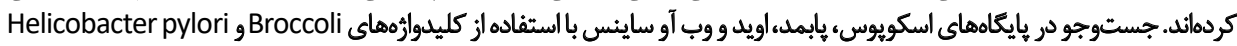
Sprouts ، H pylori،Brassica، Randomized clinical trial

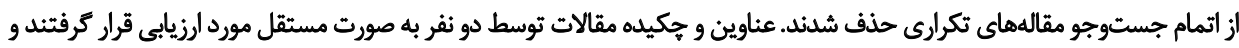

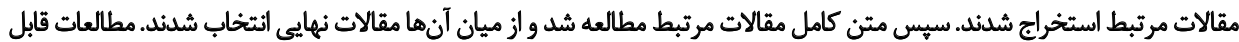

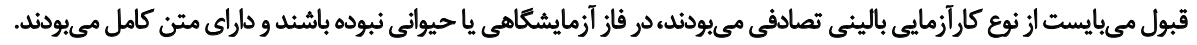

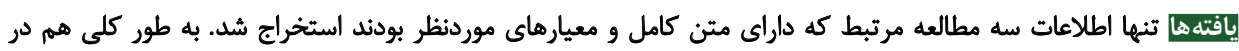

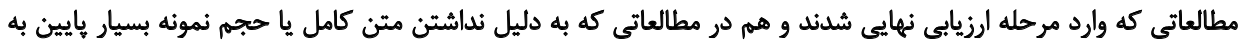

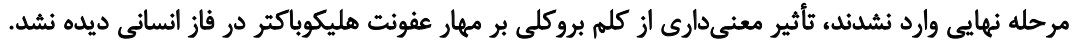

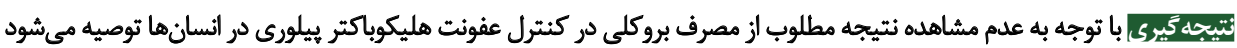

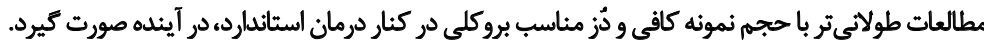

اطلاعات مقاله:

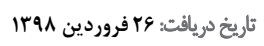

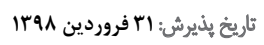
تاريخ انتشار: rا خرداد

كليدوارهها: - مها: هليكوباكتر ييلورى، هليكوباكتر، بروكلي، - مليكي، سولفوروفان، كارآزمايكي بالينى
هليكوباكتر ييلورى، يك باسيل مارييجى كرم منفى است كه

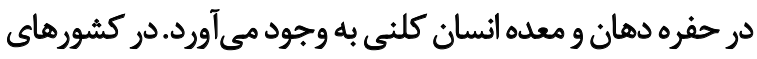

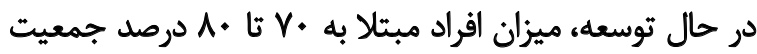

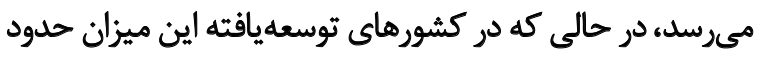

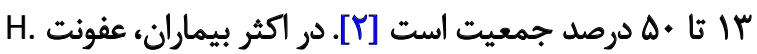

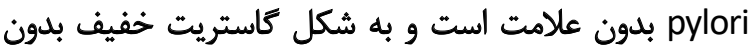

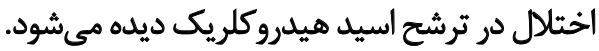

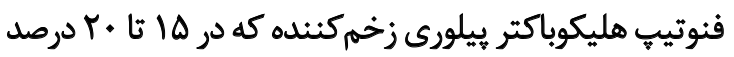

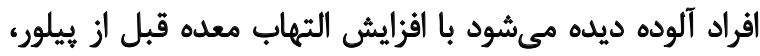

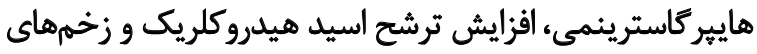

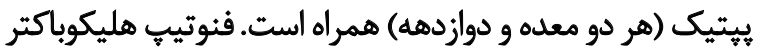

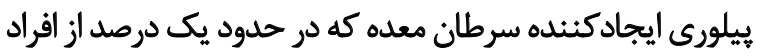

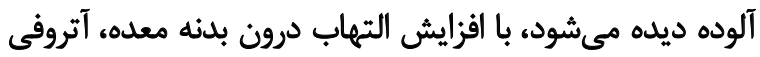

doties

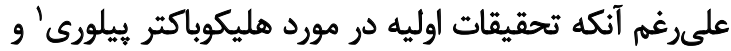

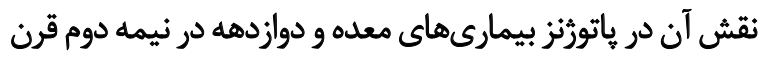

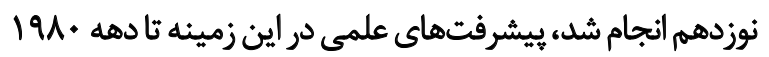

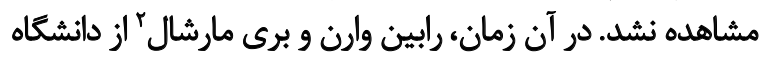

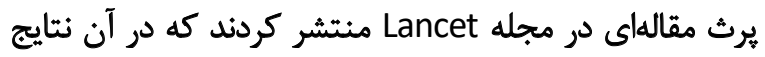

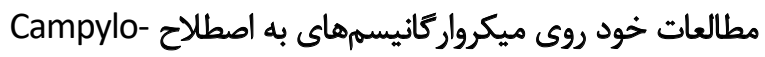

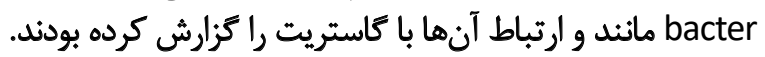

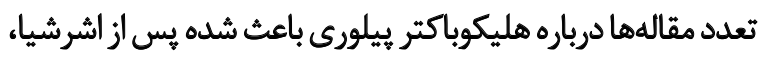
كولى بيشترين ياتوثن مورد مطالعه باشد [1]

1. H. pylori

2. Robin Warren \& Barry Marshall 
جدول ا.استراتزى جستوجوى مقالات

وازٔكان كليدى و تركيبات

1. Helicobacter pylori

2. $\mathrm{H}$ pylori

3. Brassica

4. Broccoli sprouts

5. Randomized clinical trial

6. 1 OR 2

7. 3 OR 4

8. 6 AND 7

9. 8 AND 5

وارؤان كليدى

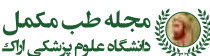

تصادفى كه تأثير كلم بروكلى را بر عفونت هليكو باكتر بيلورى جائي

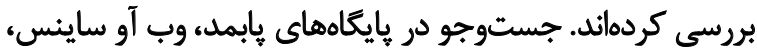

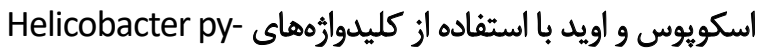
Brassica، Random- g lori، Broccoli sprouts، H pylori ized clinical trial

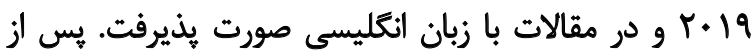

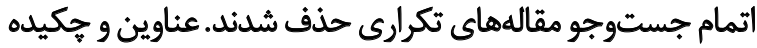

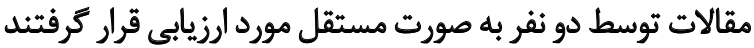
و مقالات مرثبط استخراج شدند.

مطالعات قابل قبول مىبايست از نوع كارآزمايى بالينى تصادفى

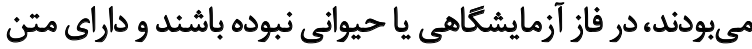

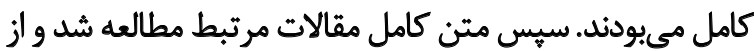

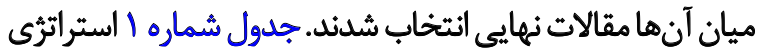

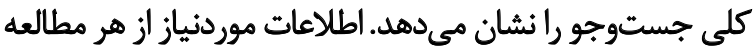

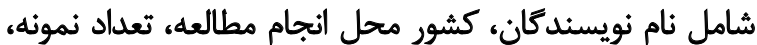

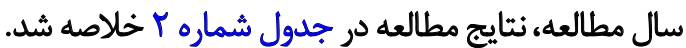

يأثتهها

در ابتدا با جستوجوى يايكاههاى اطلاعاتى و با كليدوارههاي

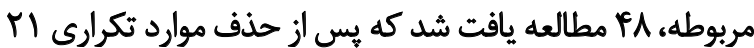

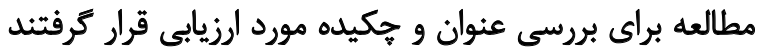

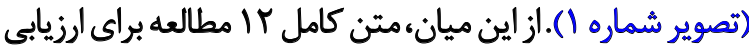

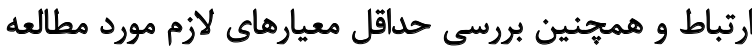

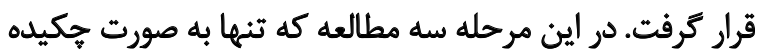

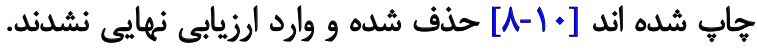

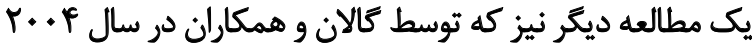

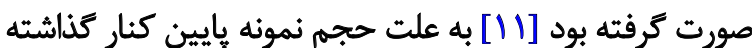

مطالعه مذكور يك مطالعه مقدماتى بود كه روى تنها 9 بيمار
غشاى مخاطى در معده قبل از ييلور، كاهش ترشح هيدروكلريك

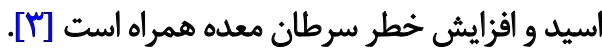

درمان فارماكولوزيك عفونت هليكوباكتر ييلورى بر اساس

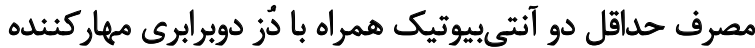

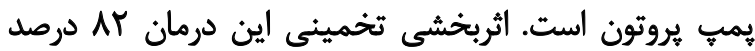

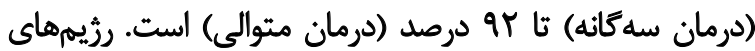

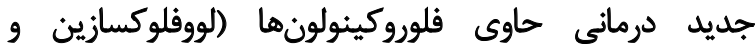

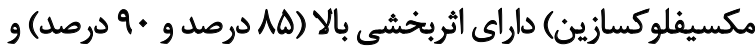

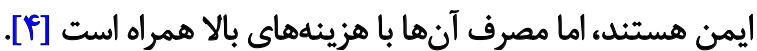

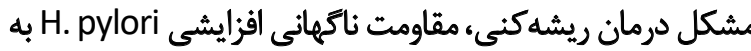
آنتىبيوتيكهاى مورداستفاده است.

به نظر ميرسد استفاده از تركيبات كياهى سبب كاهش عوارض

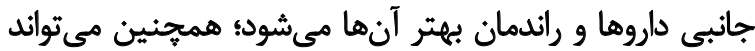

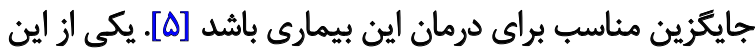

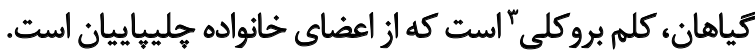

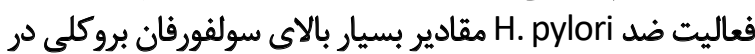

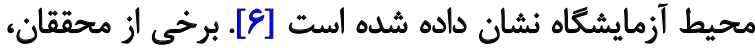

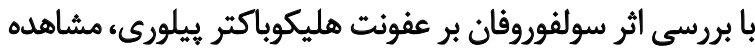

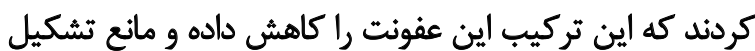

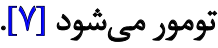

بر اساس دانش ما مطالعه مرورى نظاممندى روى اثربخشى اندي

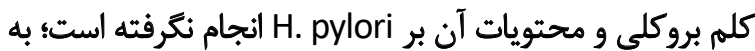

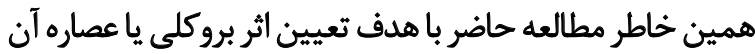
برعفونت هليكوباكتر ييلورى انجام ترفت

\section{مواد ورشها نها} اين مطالعه، مرور منظمى است بر مطالعات كارآزمايى بالينى 


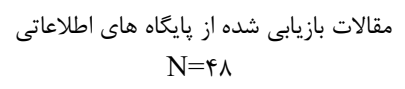

Pubmed $=6$, Web of Science $=21$, OVID $=8$, SCOPUS $=13$

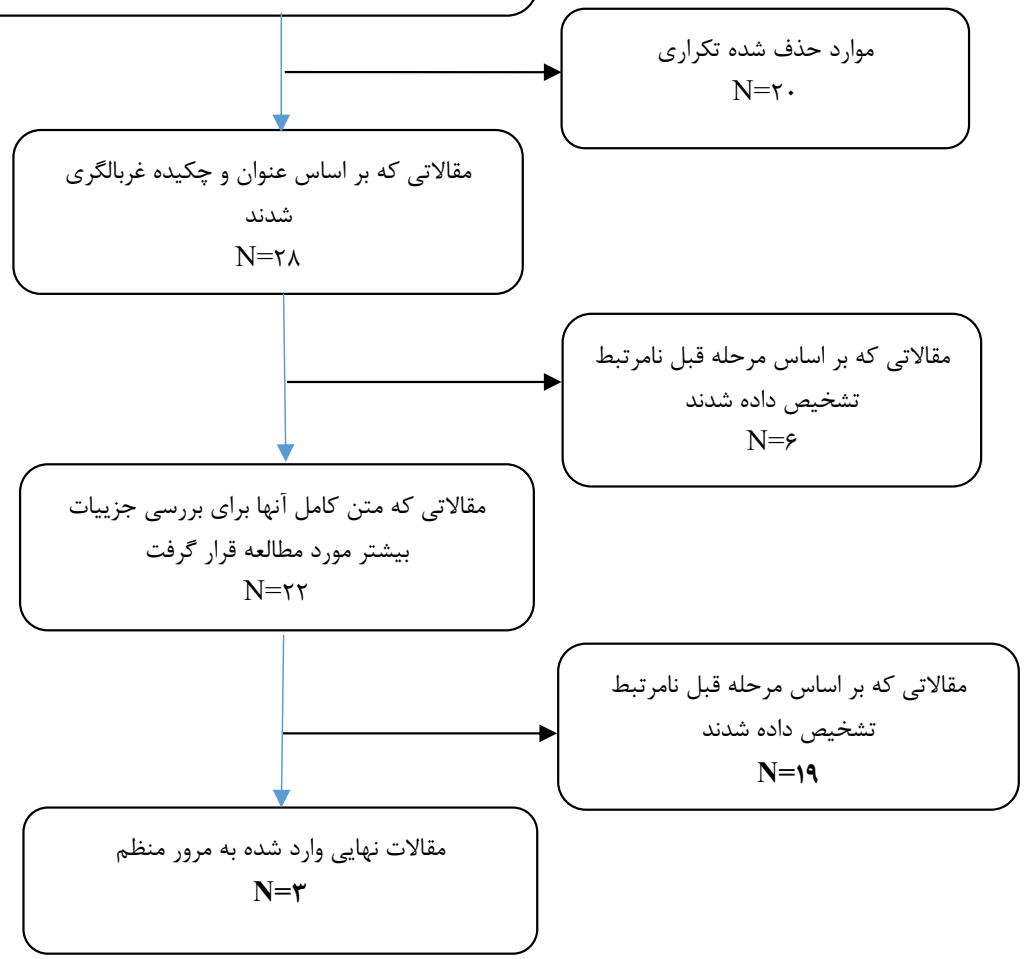

درمان توسط عصاره بروكلى حاوى سولفوروفان نتوانست تراكم

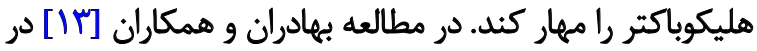

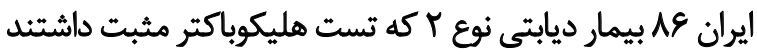

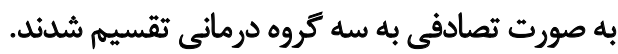

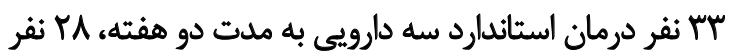

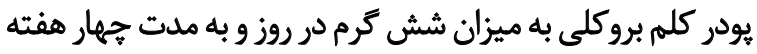

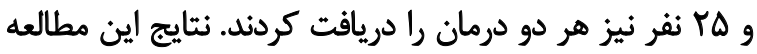

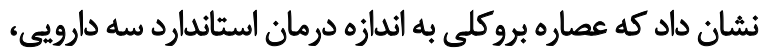

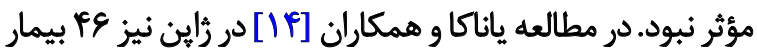

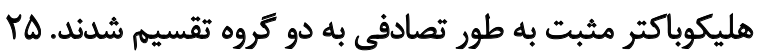

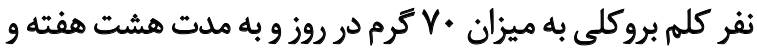

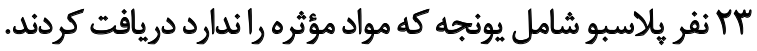

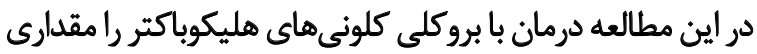

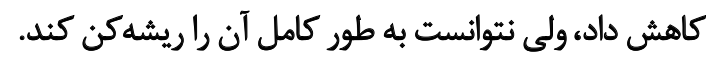
به طور كلى هم در مطالعاتى كه درنهايت وارد ارزيابى نهايى دائ

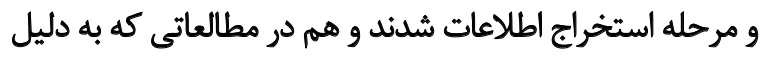

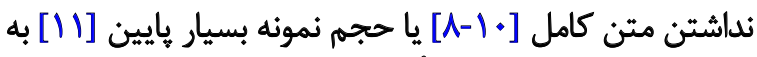

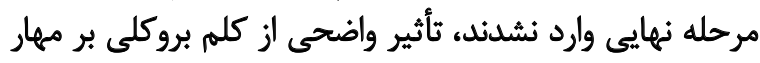

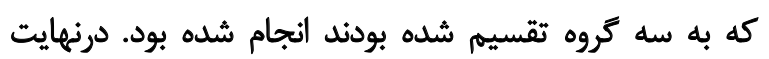

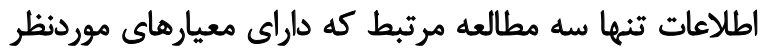

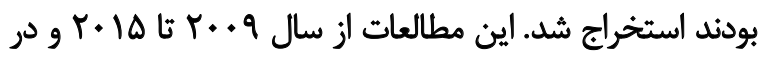

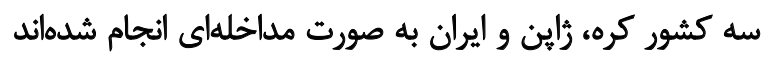

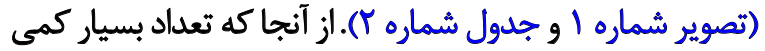

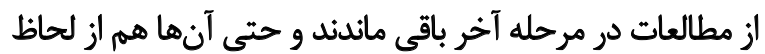

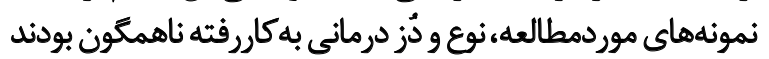

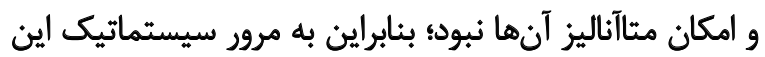
مطالعات بسنده شد (تصوير شماره ()). در مطالعه جانت و ومكاران [IT] در كره، 19 نفر به صورت

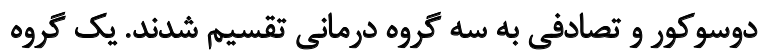

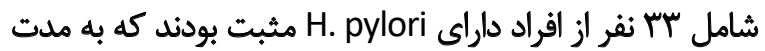

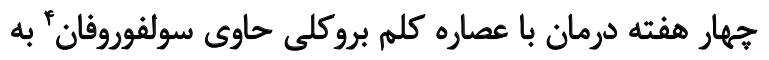

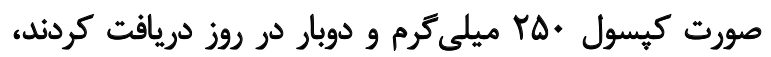

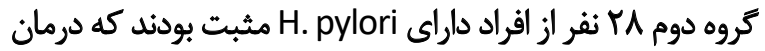

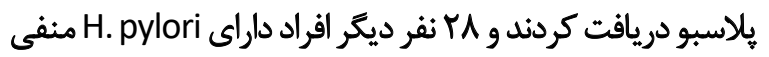

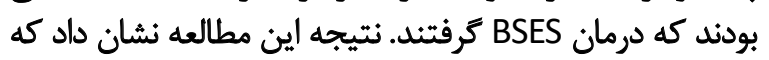

4. Broccoli Sprout Extract Containing Sulforaphane (BSES) 
جدول r. مشخصات مطالعات انجامشده در رابطه با تأثير كلم بروكلى روى عفونت هليكوباكتر

نويسندكان

\begin{tabular}{|c|c|c|}
\hline & "آ نفر H.P مثبت و & \\
\hline درمان تونسط & درمان *BSES (كيسول & 19 نفر به \\
\hline عصاره بروكلى & 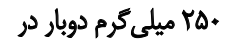 & صورت \\
\hline حاوى سولفوروفان & روز به مدت جهار هفته)، & دوسوكور و \\
\hline نثوائست تراكم & رب نفر H.P مثبت و & تصادفي به سه \\
\hline هليكوباكتر را مهار & درمان تيلاسبو و رץ نقر & كروه درماني \\
\hline كنئ & H.P منفى و درمان H.P & تقسيم شُدنل. \\
\hline
\end{tabular}

BSES

\begin{tabular}{|c|c|c|c|c|c|}
\hline 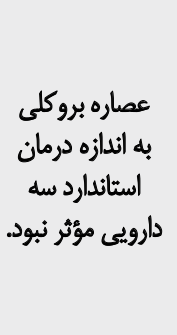 & 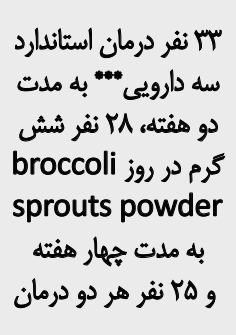 & 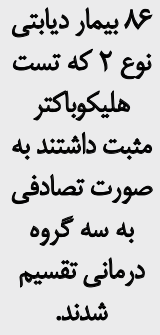 & r.lf & ايران & $\begin{array}{c}\text { Complementary and alternative } \\
\text { medicinal effects of broccoli } \\
\text { sprouts powder on Helicobacter } \\
\text { pylori eradication rate in type } 2 \\
\text { diabetic patients: A randomized } \\
\text { clinical trial }\end{array}$ \\
\hline 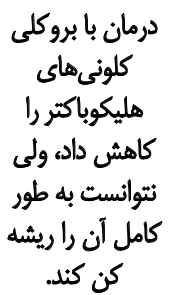 & 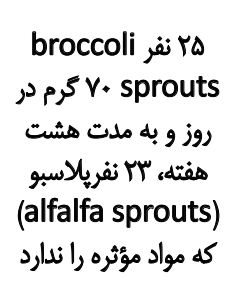 & 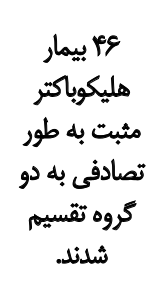 & $r+. q$ & رأين & $\begin{array}{l}\text { Dietary sulforaphane-rich } \\
\text { broccoli sprouts reduce } \\
\text { colonization and attenuate } \\
\text { gastritis in Helicobacter pylori- } \\
\text { infected mice and humans }\end{array}$ \\
\hline
\end{tabular}

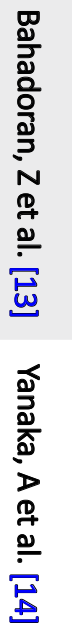

(3)

* H. pylori

** Broccoli Sprout Extract containing Sulforaphane

*** omeprazole $20 \mathrm{mg}$, clarithromycin $500 \mathrm{mg}$, amoxicillin $1000 \mathrm{mg}$, twice a day for 14 days

شد سولفوروفان مى تواند اوره را كه عامل مهمي است كه اجازه

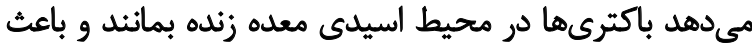

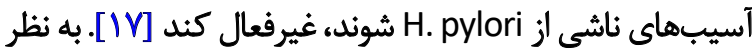

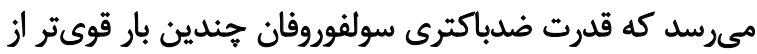

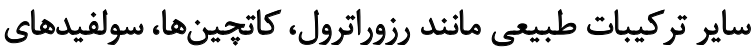

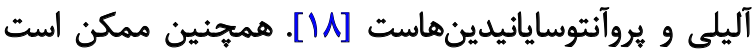

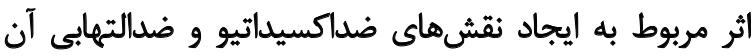

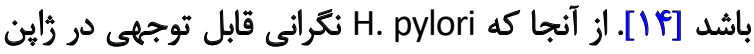

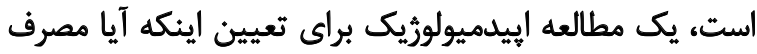

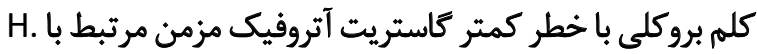
pylori

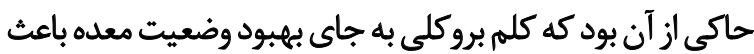

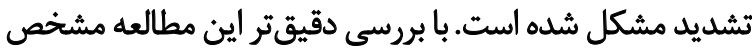

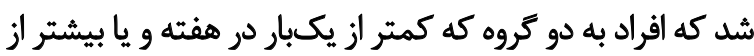

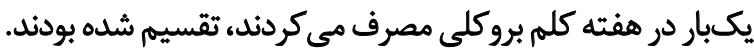

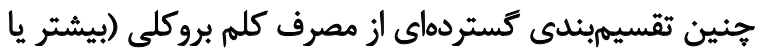

عفونت هليكوباكتر ديده نشد.

ث

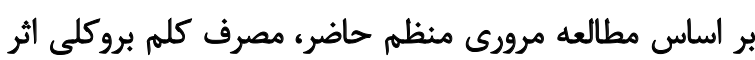

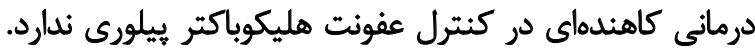

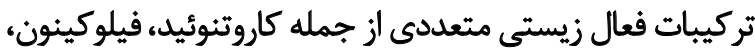

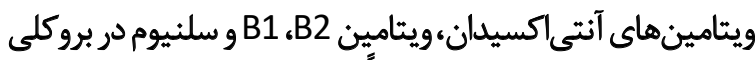

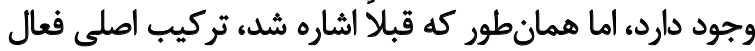

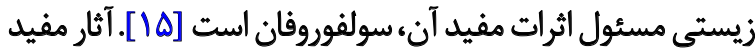

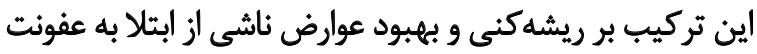

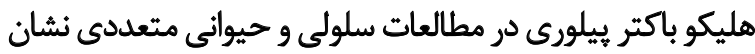

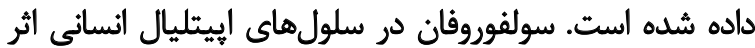

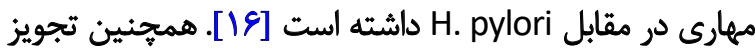

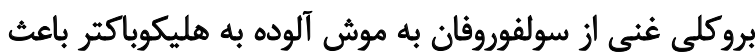

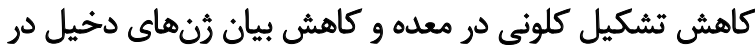

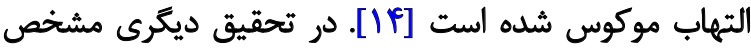


و تحليل نتايج، نوشتن مقاله و ويرايش آن شركت داشتند.

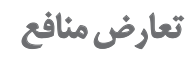

بنابـر اظهـار نويســدكان ايـن مقالـه تعـارض منافع نداشـته

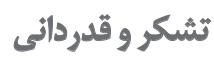

نويسندكان اين مقاله از دانشكاه علوميزشكى ارئ اراك به منظور

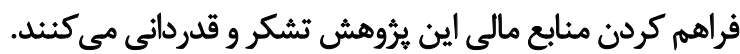

كمتر از يك وعده در هفته) حتى مى تواند شامل افرادى شود كه

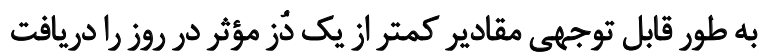

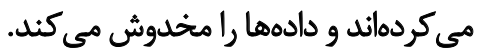

مطالعات نشان دادهاند كه تركيب ميكروبيوتاى معده (احتمالاً

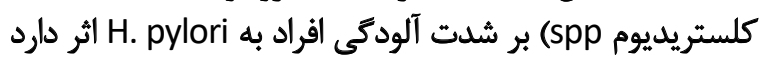

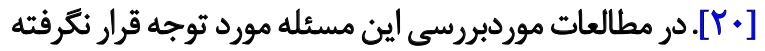

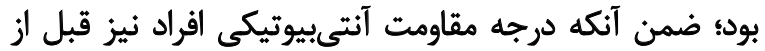

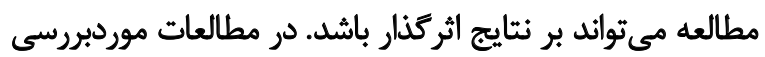

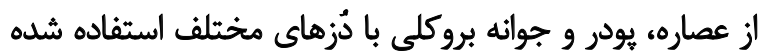

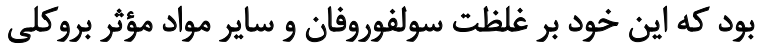

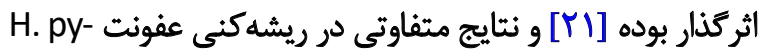
lori

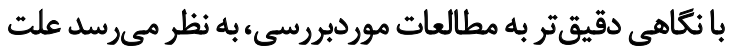

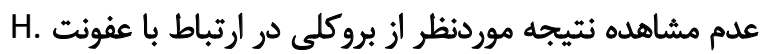

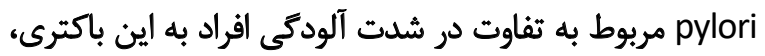

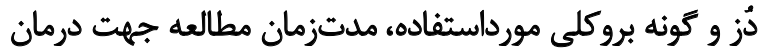

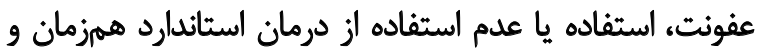
فقدان قدرت كافى مطالعه به دليل حجم نمونه ناكافى باشد.

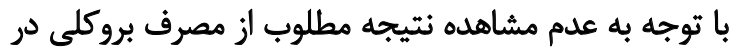

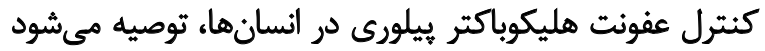

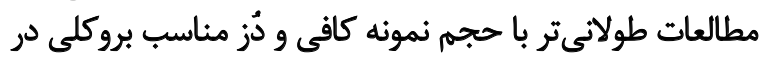
كنار درمان استاندارد در آينده صورت كيرد

تتيجه

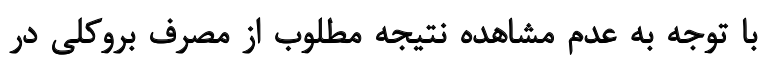

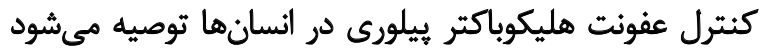

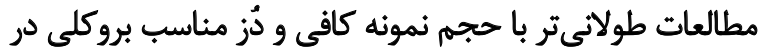

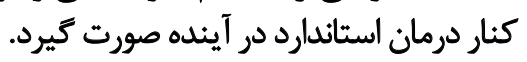

ملاحظات اخلاقى

\section{بيروى از اصول اخلاق يثوهش}

اين يك مقاله مرورى است و نمونه انسانى يا حيوانى نداشته و

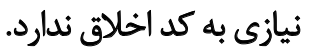

$$
\text { مامبى مالى }
$$

دانشكاه علوميزشكى اراك حامى مالى يرورثه حاضر بوده است.

$$
\text { مشاركت ثويسندكّان }
$$

فاطمه عزيزى سليمان و مريم زمانيان در همه مراحل طراحى

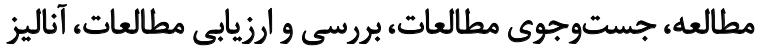




\section{References}

[1] Kidd M, Modlin IM. A century of Helicobacter pylori: Paradigms lost-paradigms regained. Digestion. 1998; 59(1):1-15. [DOI:10.1159/000007461] [PMID]

[2] Peleteiro B, Bastos A, Ferro A, Lunet N. Prevalence of Helicobacter pylori infection worldwide: A systematic review of studies with national coverage. Digestive Diseases and Sciences. 2014; 59(8):1698-709. [DOI:10.1007/s10620-014-3063-0] [PMID]

[3] Bartnik W. Clinical aspects of Helicobacter pylori infection. Polskie Archiwum Medycyny Wewnętrznej. 2008; 118(7-8):426-30. [DOI:10.20452/ pamw.440] [PMID]

[4] Li BZ, Threapleton DE, Wang JY, Xu JM, Yuan JQ, Zhang C, et al. Comparative effectiveness and tolerance of treatments for Helicobacter pylori: Systematic review and network meta-analysis. BMJ. 2015; 351:h4052. [DOI:10.1136/bmj.h4052] [PMID] [PMCID]

[5] Vítor JMB, Vale FF. Alternative therapies for Helicobacter pylori: Probiotics and phytomedicine. FEMS Immunology and Medical Microbiology. 2011; 63(2):153-64. [DOI:10.1111/j.1574-695X.2011.00865.x] [PMID]

[6] Erdogrul ÖT. Antibacterial activities of some plant extracts used in folk medicine. Pharmaceutical Biology. 2002; 40(4):269-73. [DOI:10.1076/ phbi.40.4.269.8474]

[7] Fahey JW, Zhang Y, Talalay P. Broccoli sprouts: An exceptionally rich source of inducers of enzymes that protect against chemical carcinogens. Proceedings of the National Academy of Sciences of the United States of America. 1997; 94(19):10367-72. [DOI:10.1073/pnas.94.19.10367] [PMID] [PMCID]

[8] Chang YW, Park YM, Oh CH, Oh SJ, Cho JH, Kim JW, et al. Effects of probiotics or broccoli supplementation on Helicobacter pylori eradication with standard clarithromycin-based triple therapy. The Korean Journal of Internal Medicine. 2020; 35(3):574-81. [DOI:10.3904/kjim.2019.139] [PMID] [PMCID]

[9] Jang JY. 01. Upper GI-123 (Inhibitory effect on Helicobacter pylori and antioxidant effect by broccoli sprout extract containing of sulforaphane). Journal of Gastroenterology and Hepatology. 2009; 24(s1):A30 [DOI:10.1111/j.1440-1746.2009.06083_1.x]

[10] Opekun AR, Opekun JL, Graham DY. Broccoli (Sulforaphane glucosinolate) for the treatment of $\mathrm{H}$. pylori infection in humans. Gastroenterology. 2003; 124(4 Suppl 1):A362. [DOI:10.1016/S00165085(03)81829-2]

[11] Galan MV, Kishan AA, Silverman AL. Oral broccoli sprouts for the treatment of Helicobacter pylori infection: A preliminary report. Digestive Diseases and Sciences. 2004; 49(7-8):1088-90. [DOI:10.1023/ B:DDAS.0000037792.04787.8a] [PMID]

[12] Chang YW, Jang JY, Kim YH, Kim JW, Shim JJ. The effects of broccoli sprout extract containing sulforaphane on lipid peroxidation and Helicobacter pylori infection in the gastric mucosa. Gut and Liver. 2015; 9(4):486-93. [DOI:10.5009/gnl14040] [PMID] [PMCID]

[13] Bahadoran Z, Mirmiran P, Zarif Yeganeh M, Hosseinpanah F, Zojaji H, Azizi F. Complementary and alternative medicinal effects of broccoli sprouts powder on Helicobacter pylori eradication rate in type 2 diabetic patients: A randomized clinical trial. Journal of Functional Foods. 2014; 7:390-7. [DOI:10.1016/j.jff.2014.01.020]

[14] Yanaka A, Fahey JW, Fukumoto A, Nakayama M, Inoue S, Zhang S, et al. Dietary sulforaphane-rich broccoli sprouts reduce colonization and attenuate gastritis in Helicobacter pylori-infected mice and humans. Cancer Prevention Research (Philadelphia, PA). 2009; 2(4):353-60. [DOI:10.1158/1940-6207.CAPR-08-0192] [PMID]
[15] Moreno DA Carvajal M, López-Berenguer C, García-Viguera C Chemical and biological characterisation of nutraceutical compounds of broccoli. Journal of Pharmaceutical and Biomedical Analysis. 2006; 41(5):1508-22. [DOI:10.1016/j.jpba.2006.04.003] [PMID]

[16] Fahey JW, Haristoy X, Dolan PM, Kensler TW, Scholtus I, Stephenson KK, et al. Sulforaphane inhibits extracellular, intracellular, and antibiotic resistant strains of Helicobacter pylori and prevents benzo[a]pyrene-induced stomach tumors. Proceedings of the National Academy of Sciences of the United States of America. 2002; 99(11):7610-5. [DOI:10.1073/ pnas.112203099] [PMID] [PMCID]

[17] Fahey JW, Stephenson KK, Wade KL, Talalay P. Urease from Helicobacter pylori is inactivated by sulforaphane and other isothiocyanates. Biochemical and Biophysical Research Communications. 2013; 435(1):1-7. [DOI:10.1016/j.bbrc.2013.03.126] [PMID] [PMCID]

[18] Lee SY, Shin YW, Hahm KB. Phytoceuticals: Mighty but ignored weap ons against Helicobacter pylori infection. Journal of Digestive Diseases. 2008; 9(3):129-39. [DOI:10.1111/j.1751-2980.2008.00334.x] [PMID]

[19] Sato K, Kawakami N, Ohtsu T, Tsutsumi A, Miyazaki S, Masumoto T, et al. Broccoli consumption and chronic atrophic gastritis among Japanese males: An epidemiological investigation. Acta Medica Okayama. 2004; 58(3):127-33. [DOI:10.18926/AMO/32109] [PMID]

[20] Rolig AS, Cech C, Ahler E, Elliot Carter J, Ottemann KM. The degree of Helicobacter pylori-triggered inflammation is manipulated by preinfection host microbiota. Infection and Immunity. 2013; 81(5):1382-9. [DOI:10.1128/IAI.00044-13] [PMID] [PMCID]

[21] Jeffery EH, Araya M. Physiological effects of broccoli consumption. Phytochemistry Reviews. 2009; 8:283-98. [DOI:10.1007/ s11101-008-9106-4] 
This Page Intentionally Left Blank 